\title{
Editorial
}

\section{Preoperative diagnosis of lymph node metastasis: a dream?}

\author{
John C. Lipham and Tom R. DeMeester \\ Department of Surgery, University of Southern California Keck School of Medicine, 1510 San Pablo St., Suite 514, Los Angeles, CA 90033, \\ USA
}

The authors of "Preoperative diagnosis of lymph node metastases in gastric cancer by magnetic resonance imaging with ferumoxtran-10" [1] should be commended for an excellent article. Pre-operative lymph node staging for upper GI malignancies is a difficult task. Tumor size and distant metastases are reasonably predicted preoperatively. Decisions regarding stage and appropriate therapy are, therefore, made based on the limited ability to assess lymph nodes. The results of computed tomography (CT), positron emission tomography (PET), and endoscopic ultrasound (EUS) have been disappointing with regard to sensitivity and accuracy for diagnosing lymph node metastases prior to surgery. The poor predictive value of these tests has major implications for the patient when trying to individualize treatment for the maximal curative potential and least morbidity.

The current technologies used to classify upper GI lymph node metastases rely heavily on size, shape, or metabolic activity and have not been shown to be very helpful in predicting lymph node involvement. Studies regarding PET and CT have shown poor sensitivity and accuracy in assessing lymph node status. Even when PET is combined with CT, accuracy has only been in the range of $65 \%[2,3]$. EUS has been no better in predicting lymph node involvement, with sensitivity and accuracy of $67 \%$ and $69 \%$, respectively [4]. These modalities are clearly insufficient to allow decision making on the extent of lymphadenectomy needed for an individual patient. Therefore, most patients are subjected to extended lymph node dissections regardless of actual nodal status. Some will benefit from this, but others will only incur the morbidity of radical lymphadenectomy without a survival benefit.

The decision on the method of resection also relies heavily on the lymph node status. In this day and age

Offprint requests to: J.C. Lipham with the increasing use of less-invasive surgical techniques, preoperatively determining or predicting lymph node involvement has never been more important. Patients without lymphatic spread are clearly candidates for less invasive procedures such as laparoscopic wedge resection, laparoscopic gastrectomy with less radical lymph node dissection, or even endoscopic mucosal resection. These techniques have been shown to be safe, effective, and less morbid for the patient with early disease $[5,6]$. Their application to patients with more advanced-stage cancer is not appropriate and again emphasizes the need to accurately determine the nodal status preoperatively.

Neoadjuvant chemotherapy and radiation also remains a controversial topic in regard to upper GI malignancies. Data from randomized controlled trials showing significantly improved outcomes are still scarce, and it remains uncertain which subgroups of patients may benefit from neoadjuvant therapy [7]. Frequently, patients suspected of having lymph node metastases are given pre-op chemoradiotherapy in the hopes of improving outcome. This questionably beneficial course of action carries with it substantial morbidity and is based almost entirely on the results of inadequate technology used to assess nodal status. A significant proportion of patients will therefore incur this morbidity without any potential benefit because of overestimating stage. Assuming that there is benefit to neoadjuvant therapy in those with nodal disease, preoperative determination is essential.

This technology using magnetic resonance imaging (MRI) with ferumoxtran-10 reported by Tatsumi et al. [1] may also be applicable to other GI malignancies such as esophageal and colorectal cancer. Preoperative assessment of lymph node status in these malignancies is equally difficult and clearly has similar prognostic significance. The decision regarding extent of lymph node dissection, method of resection, and the role of neoadjuvant therapy also rely heavily on the status of 
the lymph nodes. If this technology could be applied to these GI malignancies with similar sensitivity and accuracy, the ability to individualize treatment could also be improved.

The prognostic significance of lymph node status in GI malignancies cannot be overemphasized. Preoperative decisions regarding appropriate treatment are made largely on whether one believes that the tumor has spread to the lymph nodes. Clinicians frequently err on the side of overestimating the stage of a tumor because of the poor ability to accurately make this determination before surgery. The article by Tatsumi et al. [1] highlights the shortcomings of the currently available technology in assessing nodal status and offers an alternative for preoperative staging. The results of MRI with ferumoxtran-10 are encouraging with higher sensitivity and accuracy than CT, PET, or EUS. This should allow for a more selective and individualized treatment strategy for patients with gastric cancer. It is hoped that improved accuracy in preoperative staging will lead to the ability to determine the treatment with maximal curative potential and least morbidity. This is obviously the goal in the treatment of every patient with cancer.

\section{References}

1. Tatsumi $\mathrm{Y}$, Tanigawa N, Nishimura $\mathrm{H}$, Nomura E, Mabuchi $\mathrm{H}$, Matsuki M, et al. Preoperative diagnosis of lymph node metastases in gastric cancer by magnetic resonance imaging with ferumoxtran10. Gastric Cancer 2006;9:120-8.

2. Yun M, Lim JS, Noh SH, Hyung WJ, Cheong JH, Bong JK, et al. Lymph node staging of gastric cancer using (18)F-FDG PET: a comparison study with CT. J Nucl Med 2005;46:1582-8.

3. Chen J, Cheong JH, Yun MJ, Lim JS, Hyung WJ, Noh SH. Improvement in preoperative staging of gastric adenocarcinoma with positron emission tomography. Cancer 2005;103:2383-90.

4. Xi WD, Zhao C, Ren GS. Endoscopic ultrasonography in preoperative staging of gastric cancer: determination of tumor invasion depth, nodal involvement and surgical respectability. World J Gastroenterol 2003;9:254-7.

5. Lee JH, Han HS, Lee JH. A prospective randomized study comparing open vs. laparoscopy-assisted distal gastrectomy in early gastric cancer: early results. Surg Endosc 2005;19:168-73.

6. Mochiki E, Ohno T, Kamiyama Y, Aihara R, Nakabayashi T, Asao $\mathrm{T}$, et al. Laparoscopy-assisted gastrectomy for early gastric cancer in young and elderly patients. World J Surg 2005;29:1585-91.

7. Lordick F, Siewert JR. Recent advances in multimodal treatment for gastric cancer: a review. Gastric Cancer 2005;8:78-85. 